\title{
STRESS ASYMMETRY IN CYCLIC DEFORMATION OF B.C.C. METALS
}

\author{
K. J. BOWMAN ${ }^{1}$ and R. GIBALA ${ }^{2}$ \\ ${ }^{1}$ School of Materials Engineering, Purdue University, West Lafayette, IN 47907 and \\ ${ }^{2}$ Materials Science and Engineering, The University of Michigan, Ann Arbor, MI 48109, U.S.A.
}

(Received 14 February 1991)

\begin{abstract}
Asymmetric slip in b.c.c. metals is related to both the symmetry about $\frac{1}{2}\langle 111\rangle$ screw dislocations and elastic anisotropy. The elastic anisotropy differs quite substantially from one b.c.c. metal to the next and this anisotropy has been observed in measurements of asymmetric stresses in plastic deformation experiments. In this paper, data from cyclic deformation of b.c.c. metals, with special emphasis on tungsten which is elastically isotropic, are used to demonstrate the role of screw dislocation geometry and elastic anisotropy on stress asymmetry. The interaction of dislocation geometry and thermal activation of screw dislocation motion is discussed.
\end{abstract}

\begin{abstract}
Résumé-Le glissement asymétrique dans les métaux cc est lié à la fois à la symétrie autour des dislocations vis $\frac{1}{2}\langle 111\rangle$ et à l'anisotropie élastique. L'anisotropie élastique varie trés substantiellement d'un métal cc à l'autre et cette anisotropie est observée dans les mesures de contraintes asymétriques dans des expériences de déformation plastique. Dans cet article, les données provenant de la déformation cyclique de métaux $c c$, avec une attention particulière pour le tungstène qui est élastiquement isotrope, sont utilisées pour démontrer le rôle de la géométrie des dislocations vis et de l'anisotropie élastique sur l'asymétrie des contraintes. L'interaction de la géométrie des dislocations et de l'activation thermique du mouvement des dislocations vis est discutée.
\end{abstract}

\begin{abstract}
Zusammenfassung-Die Asymmetrie der Gleitung von krz. Metallen hängt sowohl mit der Symmetrie um $\frac{1}{2}\langle 111\rangle$-Schraubenversetzungen als auch mit der elastischen Anisotropie zusammen. Die elastische Anisotropie unterscheidet sich von einem zum anderen krz. Metall beträchtlich; diese Anisotropie wird bei Messungen der asymmetrischen Spannungen in Experimenten der plastischen Verformung beobachtet. In dieser Arbeit wird mit Daten zur zyklischen Verformung von krz. Metallen mit Schwerpunkt Wolfram, welches elastisch isotrop ist, die Rolle der Schraubenversetzungsgeometrie und der elastischen Anisotropie bei der Spannungsasymmetrie dargestellt. Die Wechselwirkung der Versetzungsgeometrie und der thermischen Aktivierung der Bewegung der Schraubenversetzungen wird diskutiert.
\end{abstract}

\section{INTRODUCTION}

Deformation to symmetric positive and negative plastic strains usually is expected to require symmetric tensile and compressive loads, respectively. For dislocation glide of metals the assumption that $\sigma_{\mathrm{T}}\left(\epsilon_{\mathrm{p}}\right)=\sigma_{\mathrm{C}}\left(-\epsilon_{\mathrm{p}}\right)$, where $\sigma_{\mathrm{T}}$ and $\sigma_{\mathrm{C}}$ are the tension and compression stresses at a plastic strain, $\epsilon_{\mathrm{p}}$, is often a good one. Similarly, symmetric loading levels beyond the proportional limit should produce symmetric plastic strains. Deviations from this equivalence are observed, but they are generally small unless influenced by external effects like prestrain.

In b.c.c. metals of the three-fold symmetry of $\frac{1}{2}\langle 111\rangle$ screw dislocation can result in different flow stresses for single crystals deformed to equal but opposite plastic strains [1]. At low homologous temperatures $\sigma_{\mathrm{T}}\left(\epsilon_{\mathrm{p}}\right) \neq \sigma_{\mathrm{C}}\left(-\epsilon_{\mathrm{p}}\right)$ for most orientations of b.c.c. single crystal test specimens [2]. However, the orientation relationship for tension and compression flow stresses in most b.c.c. metals does not follow predictions of an ideal orientation relationship for slip [2]. Whereas these deviations from an ideal orientation relationship have been attributed to the effects of elastic anisotropy on core dissociation of screw dislocations [3], such an ideal orientation relationship for stress asymmetry should be apparent in an elastically isotropic b.c.c. metal. In this paper the nature of slip and stress asymmetries is evaluated and compared to the geometrical consequences of slip of $\frac{1}{2}\langle 111\rangle$ dislocations in b.c.c. metals. Data from our laboratories on cyclic deformation of $\mathrm{W}$ are utilized to demonstrate that a nearly ideal orientation relationship can be obtained in an isotropic b.c.c. metal.

\section{BACKGROUND: MEASUREMENTS OF SLIP AND STRESS ASYMMETRY}

Asymmetric slip of b.c.c. metals below $\sim 0.20 T_{\mathrm{m}}$, has been demonstrated in several ways, however, the most dramatic demonstrations of slip asymmetries have been achieved in cyclic deformation. Nine $[4,5]$ has shown asymmetric plasticity during torsional fatigue of $\mathrm{Nb}$, Mo and $\mathrm{Fe}$. In symmetric load, 
fully-reversed torsion fatigue, scratches placed along the length of single crystal specimens prior to deformation become displaced around the circumference of the round bar near slip bands. The displacement of the scratches is attributed to differing amounts of plastic strain in one direction of twist versus the reverse direction. Another example of accumulated asymmetric flow is the formation of elliptical cross sections from round bars tested in push-pull fatigue. Such effects have been shown in Nb by Neumann [6] and in Fe by Mughrabi et al. [7]. The shape change of individual grains in polycrystalline $\mathrm{Fe}$ is manifested as surface roughening [8]. Somewhat earlier, slip asymmetries expressed as very large differences in flow stress, had been observed for "stubby" $\langle 100\rangle$ and $\langle 110\rangle \mathrm{W}$ single crystals stressed in alternating tension and compression by Argon and Maloof [9]. Although the experiments were conducted at relatively high plastic strain amplitudes $(\approx 4-10 \%)$, their results suggest that reversal of the deformation direction on the same crystal involves slip which is more difficult in one direction over another. In 1928, prior to the theory of dislocation glide, Taylor reported that resistance to slip in $\beta$-brass, which has a b.c.c.like crystal structure, varies with the direction of applied stress [10].

Cyclic deformation experiments in which shape change is measured usually involve the experimental difficulty of removing the extensometer from a specimen and removal of the specimen itself from the testing apparatus. Alternatively, measurements of cyclic stress asymmetry, $\Delta \sigma=\sigma_{\mathrm{T}}-\sigma_{\mathrm{C}}$, are determined by the differences in tensile $\left(\sigma_{\mathrm{T}}\right)$ and compressive $\left(\sigma_{\mathrm{C}}\right)$ peak or flow stresses at fixed plastic or total strain amplitudes. Thus, measurements of cyclic stress asymmetry can be made without interrupting the experiment to evaluate changes taking place in the specimen response. Anglada, Guiu and co-workers (AG) [11-14] have shown that measurements of $\Delta \sigma$ can be made quite readily on Mo and $\mathrm{Nb}$ by demonstrating that the level of stress asymmetry is sensitive to both plastic strain amplitude and the thermal activation parameters of temperature and strain rate. Chang et al. $[15,16]$ have reported similar findings for cyclic loading of higher purity $\mathrm{Nb}$ at rather large plastic strain amplitudes (2-4\%).

Because the dramatic changes in specimen morphology noted above for torsional and push-pull fatigue occur at homologous temperatures greater than $0.10 T_{\mathrm{m}}$ [4-8], the implication is that shape changes are not dependent on thermal activation in the same way as are monotonic flow stresses, cyclic saturation stresses or cyclic stress asymmetry. Results for push-pull fatigue of $\mathrm{Fe}$ at room temperature $\left(T / T_{\mathrm{m}}=0.16\right)$ support this conclusion since dramatic shape changes occur in the absence of measurable cyclic stress asymmetry [8].

The orientation dependence of cyclic stress asymmetry discussed by $A G$ and noted in reviews by Christian [2] and Vitek [17] does behave consistently

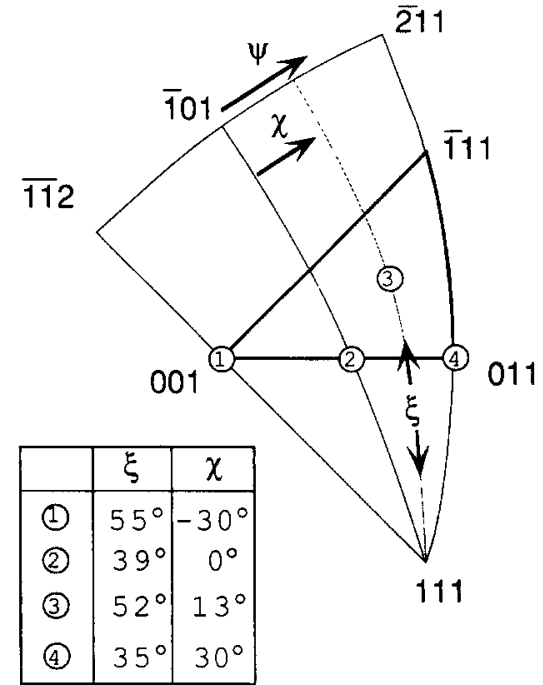

Fig. 1. Definition of $\xi, \chi$ and $\psi$ for orientation stereogram and the orientations used in this investigation.

with an increased difficulty for screw dislocation motion at lower homologous temperatures. However, when compared to ideal the ideal orientation relationship for screw dislocation geometry, termed twinning/anti-twinning asymmetry (TATA), stress asymmetries are typically shifted in the compressive sense. Conditions of ideal TATA are met when

$$
\Delta \sigma(\chi)=-\Delta \sigma(-\chi)
$$

where $\Delta \sigma$ is stress asymmetry as defined above and the angle $\chi$ is defined in Fig. 1. The angle $\chi$ is the normal to the plane of maximum shear for the $\frac{1}{2}$ [111] Burgers vector. Orientations near the center of the stereographic triangle should produce no stress asymmetry $(\Delta \sigma=0)$ for ideal TATA behavior. Yet, large negative (compressive) values of stress asymmetry are observed both by $\mathrm{AG}$ [11-14] and Chang et al. $[15,16]$. Duesbery $[18,19]$ and Vitek $[17,20]$ have attributed deviations from TATA to the effect of non-glide shear stresses on the screw dislocation core transformations which take place to allow screw dislocation motion. The non-glide shear stress is related to elastic anisotropy as it arises from the loss of spherical symmetry in elastic properties if the material is not isotropic.

\section{TWINNING/ANTI-TWINNING ASYMMETRY}

As mentioned above, the origin of TATA arises from the combination of b.c.c. crystal symmetry of $\frac{1}{2}\langle 111\rangle$ screw dislocations and the test specimen symmetry. Cyclic deformation provides an opportunity to investigate stress asymmetry effects after substantial cyclic hardening has given way to a state of cyclic saturation. Nonetheless, the retention of initially asymmetric characteristics from crystal growth or the development of asymmetric dislocation networks might be possible considering the regularity 
of dislocation networks at cyclic saturation. Duesbery $[18,19]$ has described the possible influence of deviations from crystal symmetry produced by physical dislocation of a crystal. The only nonconformity to TATA likely in ideal, pure b.c.c. single crystals results from atomic structure and bonding. Since the nature of nearest and next nearest neighbor ionic interactions are primarily responsible for elastic constants, elastic anisotropy arises from crystal structure and the character of bonding. The following discussion provides the groundwork for later descriptions of the role of elastic anisotropy in deviations from ideal TATA.

In b.c.c. crystals the twinning and anti-twinning directions of dislocation motion come about the stacking sequence of $\{112\}$ planes. Shown in Fig. 2 are (1I0) and (II2) projections demonstrating the stacking of atoms in $\{112\}$ planes which have a repeat distance of six atomic layers. Hard ball models for a perfect crystal, a crystal dislocated in the twinning sense and a crystal dislocated in the anti-twinning sense are shown. By shearing the perfect crystal in the $\frac{1}{6}[111]$ direction for all layers above $D$, a crystal dislocated in the twinning sense is produced. A twinned structure results if layers $E$ through $A$ are dislocated in the same manner. Translation of layers in the opposite direction, $\frac{1}{6}[\overline{1} \overline{1} \overline{1}]$, yields an unstable configuration. The close approach of atoms produced between layers $D$ and $E$ is the result of dislocating the crystal in the highly unlikely anti-twinning sense. To determine whether or not a particular $\langle 111\rangle$ is in the twinning direction for a given $\{112\}$ note that the twinning planes are those in which the $\langle 100\rangle$ direction of the same zone lies between the particular $\langle 111\rangle$ and the $\{112\}$ normal (as in Fig. 2).

The description of TATA given here is not intended to suggest that the motion of both edge and screw dislocations will always be asymmetric. The asymmetry is only for slip on $\{112\}$ under conditions in which significant thermal activation is necessary for screw dislocation core transformations. Because the core structure of screw dislocations is dissociated into a position between $\{112\}$ and $\{110\}$, the core transformations which must take place to accomplish motion of screw dislocations depend on TATA even though the atomic translations of the screw dislocation take place on $\{110\}$. Edge dislocation cores are not dissociated onto $\{112\}$ and therefore are not constrained to follow TATA behavior when operating on $\{110\}$. Slip of edge dislocations on $\{112\}$ may also contribute to a differential between twinningand anti-twinning, but the planar nature of edge dislocations should minimize the difference between two opposite directions of slip. This has been demonstrated in experiments at relatively high homologous temperatures and low plastic strain amplitudes where the contribution of edge dislocation motion to flow is large [3, 11-14].

The screw dislocation core is superimposed over the step produced as the dislocation moves on a slip plane in the direction corresponding to tensile deformation as illustrated in Fig. 3. The case shown is that for a single crystal of center-triangle orientation $\chi \approx 15$. For the relaxed screw dislocation core the three nodes are each displaced by $\frac{1}{6}[111]$ to form a counter-clockwise spiral out of the page in the [111] direction. Each node, termed a fractional dislocation to distinguish them from partial dislocations, represents the positional shift of the atoms along the screw dislocation core. The nodes fall near $\{110\}$ with an asymmetric extension towards a particular $\{112\}$. Thus, the three columns of atoms adjacent to the core fall between $\{110\}$ and $\{112\}$. The sense of this relaxation can take on either configuration shown with the relaxation towards the $\{112\}$ plane either leading as in Fig. 3 or following the $\{110\}$ planes along the screw spiral [1]. If this screw dislocation core configuration is sheared such that the dislocation helix tends towards the $\{112\}$ plane adjacent to the core relaxation, the material is sheared in the soft or twinning sense and dislocations are easy to move. If the core configuration is sheared in the opposite sense, then an unstable, high energy configuration of the type displayed in Fig. 2 is an intermediate step prior to motion of the screw dislocation. A shear of this type is in the hard or anti-twinning sense. In this instance it will be easier to move the pair to the left, such that compressive deformation will be easier than tensile deformation as expected for negative values of $\chi$. The barrier to dislocation motion in either case is the local core rearrangements which must take place to move the dislocation one unit translation.

The orientation relationship of TATA for a $\frac{1}{2}[111]$ screw dislocation is given in Fig. 4. The core configurations are shown for the $\chi=0^{\circ}$ and $\pm 30^{\circ}$ orientations for specimens with the trace of the deformation axis vertical on the page. The right set of core configurations, b,d,f, are equivalent sets of core configurations with the same Burgers vector but the opposite polarity to the left set, a,c.e [1]. For the core configurations $b, d, f$ the extension of the screw core towards twinning $\{112\}$ follows rather than leads the $\{110\}$ along the screw spiral. Together each set of core configurations is viewed in the [ $[\overline{1} \overline{1}]$ corresponding with different specimen orientations.

Although there are intermediate steps which lie between the two core configurations for moving dislocations, the core of an individual screw dislocation alternates between the two configurations given in Fig. 4 during the transformations required to produce motion of the dislocation from one Peierls valley to the next [2]. Thus, the more difficult of the two core transformations controls screw dislocation motion. The relationship between the two configurations for $\chi=+30^{\circ}$ and $\chi=-30^{\circ}$ reveals that the two pairs of core configurations at these orientations are simple opposites with respect to the direction of dislocation motion. The resistance to flow in tension at $\chi=+30$ and compression at $\chi=-30^{\circ}$ should 


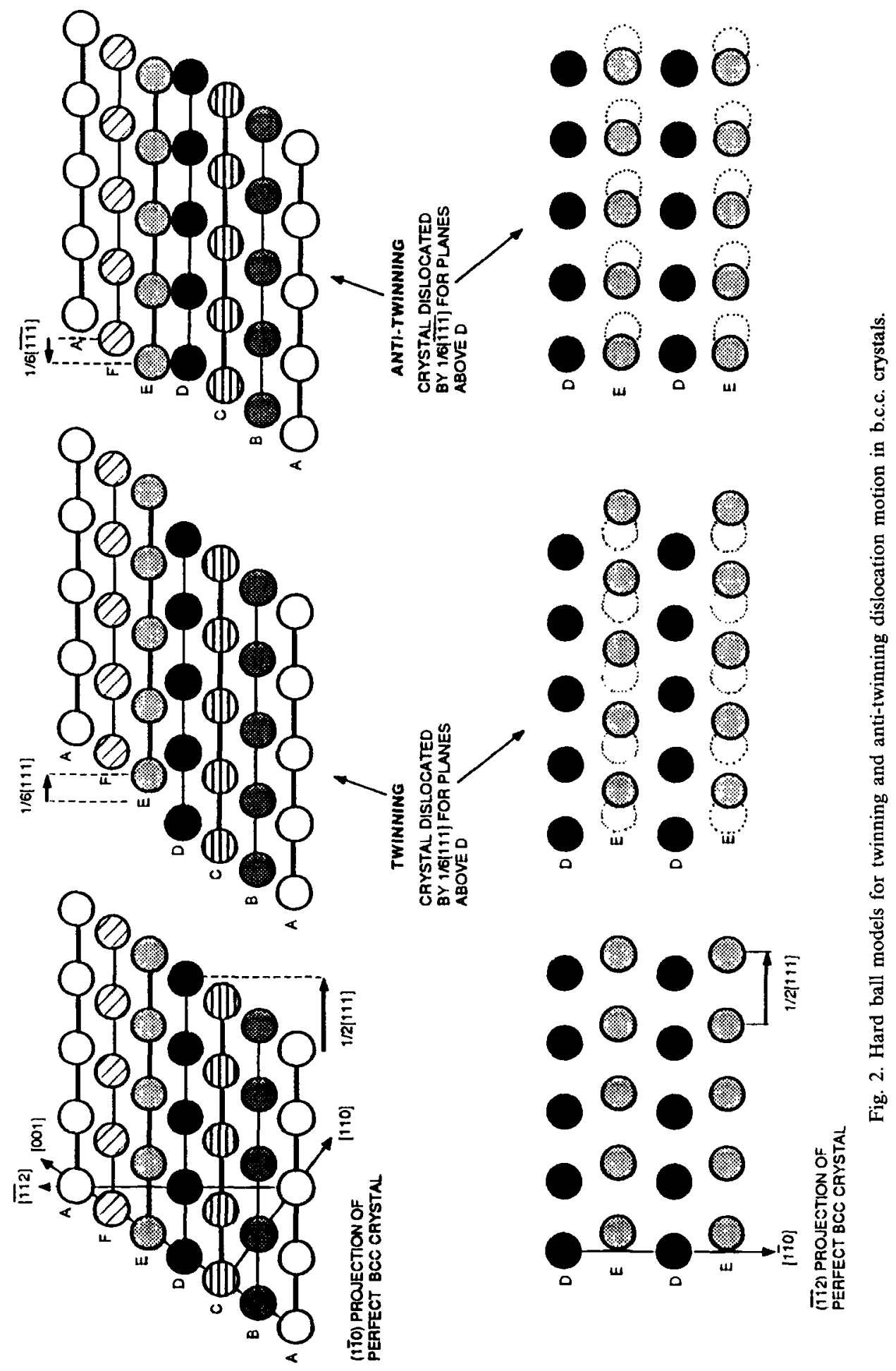



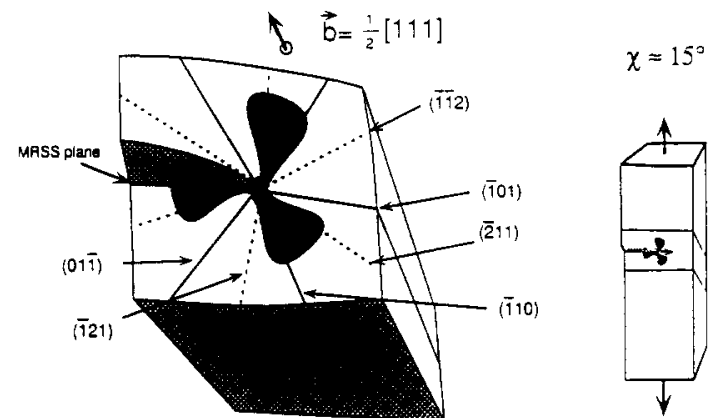

Fig. 3. Schematic drawing of the core of a $\frac{1}{2}$ [111] dislocation superimposed over the step left behind during passage of a screw dislocation on the MRSS plane.

be equal in magnitude. The reverse is also true, giving

$$
\begin{aligned}
\sigma_{\mathrm{C}}\left(+30^{\circ}\right)=\sigma_{\mathrm{T}}\left(-30^{\circ}\right) & \\
& <\sigma_{\mathrm{T}}\left(+30^{\circ}\right)=\sigma_{\mathrm{C}}\left(-30^{\circ}\right)
\end{aligned}
$$

where $\sigma_{\mathrm{T}}$ and $\sigma_{\mathrm{C}}$ coincide with the tensile and compressive stresses imparting the shear stresses required to move the dislocations shown as oriented in Fig. 4. Values of stress asymmetry, $\Delta \sigma$, for arbitrary stress levels at the orientations $\chi= \pm 30^{\circ}$ and $0^{\circ}$ gives the behavior shown in Fig. 5(a). Thereby coinciding with the expectations for a maximum resolved shear stress (MRSS) criterion, $\psi=\chi$ as shown in Fig. 5(b).

The evaluation of slip and stress asymmetry presented in this paper assumes a steady-state dislocation structure is produced during cyclic deformation. In this instance, the only relevant effect on

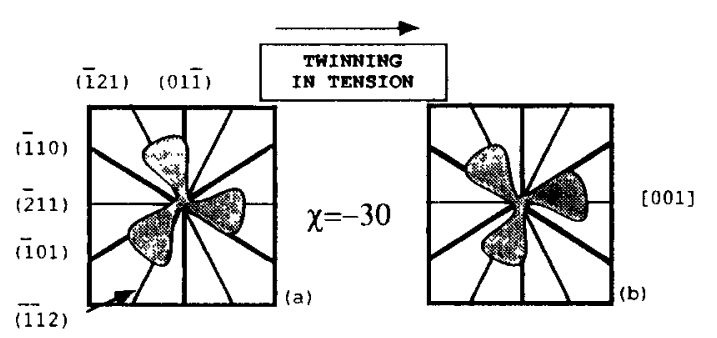

(101)

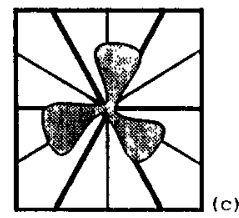

$\chi=0$
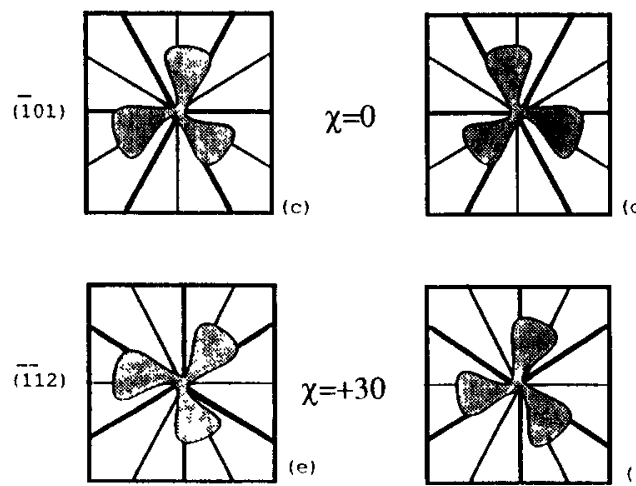

ANTI - TKINHING

IN TENSION

Fig. 4. Orientation dependence of TATA for different screw dislocation core configurations

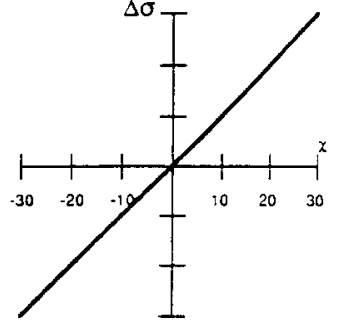

(a)

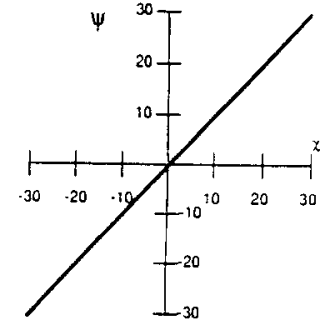

(b)
Fig. 5. (a) $\Delta \sigma$ vs $\chi$ and (b) $\psi$ vs $\chi$ for ideal b.c.c. crystals.

dislocation motion imposed by cyclic deformation experiments should be that of normal stress effects on dislocation mobility. The asymmetric slip exhibited should only arise from features intrinsic to the particular crystal structure and interatomic bonding. Of course, this is only true if the steady-state dislocation structures arising from cyclic deformation form in a symmetric manner, an assumption that relies on symmetric activity of dislocation sources in tension and compression. Experiments in simple shear [21] have successfully related slip asymmetry in deformation of $\mathrm{Nb}$ on $\{110\}$ and $\{112\}$ planes, but such experiments are difficult to perform and evaluate.

The elastic properties of b.c.c. metals differ from one metal to another, but the elastic constants of the Group V and VI transition metals are generally much more isotropic than other cubic metals. The two b.c.c. metals for which cyclic stress asymmetries have previously been investigated in detail, $\mathrm{Nb}$ and $\mathrm{Mo}$, have a similar elastic anisotropy as defined by the compliance anisotropy factor

$$
J=s_{11}^{\prime}-s_{12}^{\prime}-\frac{1}{2} s_{44}^{\prime}
$$

where the $s_{i j}$ are elastic compliance constants [1]. Ta $(J=0.338)$ and $\mathrm{Fe}(J=0.601)$ have inverse values of $J$ from $\mathrm{Nb}(J=-0.85)$ and Mo $(J=-0.102)$. W $(J=0)$ is almost perfectly isotropic over a wide range of temperatures. The value of $J$ for $\mathrm{W}$ varies from -0.01 to 0.02 over a temperature range of 77 to $500 \mathrm{~K}[22,23]$. Cubic materials with $J<0$, e.g. $\mathrm{Nb}$ and $\mathrm{Mo}$, are manifested by a higher modulus in the [100] direction and a lower modulus near the [011]-[111] boundary of the stereographic triangle. For $J>0$, e.g. $\mathrm{Ta}$ and $\mathrm{Fe}$, the inverse is true; the Young's modulus is higher near the [011]-[111] directions. For $J=0$, e.g. W, the modulus is the same in all directions.

The symmetry of a crystal is reduced by the presence of a single screw dislocation [18]. The elastic displacement field about a screw dislocation for a b.c.c. metal with anisotropic elastic constants will differ about $\langle 111\rangle$ over the elastic displacement field for a b.c.c. metal with isotropic elastic constants. Thus, the soft or hard transformations of screw dislocation cores under applied stress are superimposed upon the orientation dependence of elastic properties and specimen symmetry. For $J<0$ vs $J>0$ the superposition of the reduced symmetry 
elastic displacement field over the relaxed core configurations coincide with an opposite sign for the non-glide shear stresses [18]. Unusual features of elastic anisotropy in b.c.c. crystals have been discussed by Milstien and Marshall [24]. Although the macroscopic elastic constants are not affected greatly by alloying, the interference of solute atoms with screw dislocation core transformations may affect the orientation dependence of mechanical behavior in b.c.c. metals [25].

\section{EXPERIMENTAL RESULTS AND DISCUSSION}

Slip asymmetry was originally expressed [26] in terms of visible slip and the changes in lattice rotation with deformation. Evaluations of asymmetric slip behavior are often expressed in terms of $\psi-\chi$ curves [Fig. 5(b)] to relate deviations of slip from the MRSS criterion as shown by Taylor [26]. The acknowledged deviation of b.c.c. metals from the Schmid law is often described concurrently with it's use to report shear stresses for deformation. Because deviations from the Schmid law vary with experimental conditions and from one b.c.c. metal to another (see earlier discussion), comparison of shear stresses can be rather ambiguous. For this reason, the data given here are expressed only as the normal stress. Stress asymmetry, $\Delta \sigma$, is reported in terms of normal stresses where $\Delta \sigma=\sigma_{\mathrm{T}}-\sigma_{\mathrm{C}}$, as described above. Where data reported in shear stresses, $\tau$, are cited a conversion factor of $\Delta \sigma=2 \Delta \tau$ is used. Each set of data given is for cyclic deformation tests carried out at fixed strain limits on single crystals grown and purified by zone melting. The results for $\mathrm{W}$ are for crystals which also were heat treated at $2800 \mathrm{~K}$ for $60 \mathrm{~h}$ at a vacuum better than $1.3 \times 10^{-7} \mathrm{~Pa}$ [3].

The relationship between $\Delta \sigma$ and $\chi$ for varying plastic strain levels in incremental step tests at a strain rate of $2 \times 10^{-4}$ is given for $\mathrm{W}$ in Fig. 6. At orientation 1 (as defined in Fig. 1) an initially small stress asymmetry increases steadily to a value of near $-100 \mathrm{MPa}, \Delta \sigma \approx 0.10 \sigma_{\mathrm{C}}$, at a plastic strain amplitude, $\epsilon_{\mathrm{pl}}=3.75 \times 10^{-3}$. For orientation 2 no significant stress asymmetry, $\Delta \sigma< \pm 0.005 \sigma_{\mathrm{C}}$, was measured at any $\epsilon_{\mathrm{pl}}$. For orientation 3 the stress

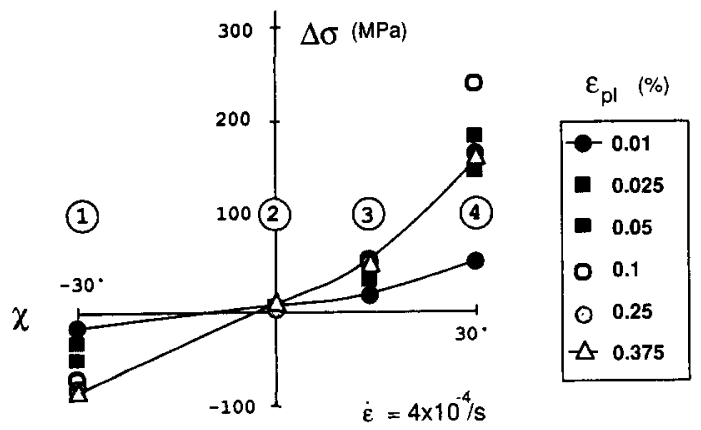

Fig. 6. $\Delta \sigma$ at several levels of $\epsilon_{\mathrm{pl}}$ vs $\chi$ for W from incremental step tests.



Fig. 7. $\Delta \sigma / E$ at several levels of $\epsilon_{\mathrm{pl}}$ vs $\chi$ for $\mathrm{W}$ and other b.c.c. metals over a range of homologous temperatures.

asymmetry increased initially and peaked at a value of approximately $25 \mathrm{MPa}, \Delta \sigma \approx 0.03 \sigma_{\mathrm{T}}$. The stress asymmetry at orientation 4 increased rapidly, reaching a maximum of $230 \mathrm{MPa}, \Delta \sigma \approx 0.23 \sigma_{\mathrm{T}}$, at $\epsilon_{\mathrm{pl}}=1 \times 10^{-3}$. At larger plastic strain amplitudes the stress asymmetry decreased to a value of $170 \mathrm{MPa}$ at $\epsilon_{\mathrm{pl}}=3.75 \times 10^{-3}$. The stress asymmetry measured at orientations 3 and 4 are exaggerated by a compressive yield phenomenon that arises before the stress asymmetry is recorded at a fixed $\epsilon_{\mathrm{pl}}$ [3].

To compare the cyclic stress asymmetry behavior of $\mathrm{W}[3,27]$ to results available for other b.c.c. metals, values of $\Delta \sigma$ normalized by Young's modulus versus $\chi$ are plotted in Fig. 7 for single level tests on $\mathrm{W}$ along with those of $\mathrm{Nb}$ and $\mathrm{Mo}$ from other investigations [11-16]. The value of Young's modulus used for normalization was the average value normally given for polycrystals. From this figure it is apparent that the orientation dependence of $\Delta \sigma$ observed in $W$ is very different than that observed for $\mathrm{Nb}$ and $\mathrm{Mo}$. When a maximum value of $\Delta \sigma$ is employed rather than $\Delta \sigma$ at $\pm \epsilon_{\mathrm{p}}$ as given above $[3,27]$, data for $\mathrm{W}$ suggest a nearly perfect antisymmetric relationship $[\Delta \sigma(\chi)=-\Delta \sigma(-\chi)]$. The data for macroscopic plastic strain amplitudes taken from single level experiments and incremental step cyclic deformation experiments on $\mathbf{W}[3,27]$ both fit ideal TATA behavior quite well relative to the other b.c.c. metals. 
Additionally, the relative homologous temperature and strain rate conditions for the normalized values of cyclic stress asymmetry given in Fig. 7 correspond to the temperature and strain rate dependence of cyclic stress-strain behavior in b.c.c. metals, i.e. cyclic stress asymmetry increases with decreasing temperature and increasing strain rate. At low temperatures, high strain rates and high plastic strain levels, the large contribution of difficult screw dislocation motion is responsible for high stress levels. The values of $\Delta \sigma / E$ shown in Fig. 7 are expected to be dependent on all three of these conditions.

A compelling case for the relevance of elastic anisotropy can be made when the normalized cyclic stress asymmetries of two b.c.c. metals like $\mathrm{Nb}$ and Mo, which have the same type of elastic anisotropy, are so similar. The stress asymmetry for $\mathrm{Fe}$, a material with an inverse elastic anisotropy from $\mathrm{Nb}$ and Mo, suggests a different cyclic stress asymmetry [28]. If the ideal TATA behavior of $W$ exhibited in this investigation is a manifestation of elastic isotropy, then it follows that non-ideal behavior of anisotropic b.c.c. metals should be a consequence of elastic anisotropy. As discussed earlier, elastic anisotropy is manifested as an orientation dependence of elastic behavior. This orientation dependence should be symmetrically reflected in the deviations of stress asymmetry from ideal TATA behavior. Because the elastic anisotropy of $\mathrm{Ta}$ and $\mathrm{Fe}$ is the inverse of that observed in $\mathrm{Nb}$ and $\mathrm{Mo}$, the cyclic stress asymmetry behavior of Ta and Fe could be anticipated to be inverse, or at least very different than that observed in $\mathrm{Nb}$ and $\mathrm{Mo}$. The results on $\mathrm{Fe}$ by Kim et al. [28] suggest high purity Fe may follow this prediction, although the experiments were not directed towards the problem of stress asymmetry. To date, experimental verification is not available in the form of cyclic stress asymmetry measurements for $\mathrm{Ta}$ and $\mathrm{Fe}$ at low homologous temperatures.

Monotonic experiments from several investigations on Ta suggest, at least at conditions under which anomalous slip is not observed, a stress asymmetry such that flow stresses in tension are slightly greater than those in compression at $\chi \approx 0$ orientations [2, 25, 29-31]. Unfortunately, experiments on the same materials in monotonic and cyclic deformation may produce stress asymmetries which are very different in magnitude, or even of the opposite sign due to differences in test geometry and specimen shape. Without comparable cyclic deformation data on a b.c.c. metal with $J>0$, the above predictions of stress asymmetry remain unproven.

Another effect of screw dislocation geometry in b.c.c. metals is the relative ease of edge dislocation versus screw dislocation motion at low homologous temperatures [2]. As shown elsewhere [32] dislocation structures from monotonic and cyclic deformation of b.c.c. metals deformed at low temperatures are determined by the mobilities of edge and screw dislocations. Yet, an apparent difficulty in maintaining production of edge dislocations leads to a dominance of screw dislocations in the dislocation dynamics of b.c.c. metals deformed to macroscopic strains at low homologous temperatures. Since little or no stress asymmetry is observed at low $\epsilon_{\mathrm{pl}}$ conditions (Fig. 6) wherein cyclic deformation is dominated by edge dislocations, the tie between screw dislocations and stress asymmetry is clearly demonstrated.

Although details of the specific screw dislocation transformations which take place during plastic deformation have not been determined with any certainty, primary relationships between crystal symmetry, dislocation geometry and elastic anisotropy are demonstrated in the cyclic deformation behavior of b.c.c. metals. The relative importance of other factors like the temperature and strain rate dependence of cyclic stress asymmetry have not been determined in a quantitative fashion, even though substantial efforts have been made to determine the ideal temperature and strain rate behavior of b.c.c. metals using cyclic deformation [33-35]. Future work on the temperature and strain rate dependence of stress asymmetry as a function of the orientation and elastic anisotropy of the crystal would definitely improve the understanding of mechanical behavior in b.c.c. metals.

Acknowledgements - This work was supported by the National Science Foundation under Grant No. DMR-8506705 and DMR-8810058. The authors would like to thank G. L. Liedl for his helpful comments on this manuscript.

\section{REFERENCES}

1. J. P. Hirth and J. Lothe. Theory of Dislocations. 2nd edn, pp. 370-371. Wiley, New York (1982).

2. J. W. Christian, Metall. Trans. 14A, 1237 (1983).

3. K. J. Bowman, Ph.D. thesis, Univ. of Michigan (1987).

4. H. D. Nine, Phil. Mag. 26A, 1409 (1972).

5. H. D. Nine, J. appl. Phys. 44, 4875 (1973)

6. R. Neumann, Z. Metallk. 66, 26 (1975).

7. H. Mughrabi and C. Wüthrich, Phil. Mag. 33A, 963 (1976).

8. H. Mughrabi, K. Herz and X. Stark. Int. J. Fract. 17, $193(1981)$

9. A. S. Argon and S. R. Maloof, Acta metall. 14, 449 (1966).

10. G. I. Taylor, Roy: Soc. Proc. 118A, I (1928).

11. M. Anglada, B. Etemad, J. A. Planell and F. Guiu, Scripta metall. 14, 1319 (1980).

12. M. Anglada and F. Guiu, Proc. Fifth Int. Conf. on Strength of Metals and Alloys, pp. 1237-1242. Pergamon Press, Oxford (1979).

13. M. Anglada and F. Guiu, Phil. Mag. 44A, 499 (1981).

14. M. Anglada and F. Guiu, Phil. Mag. 44A, 523 (1981).

15. L. N. Chang, G. Taylor and J. W. Christian, Scripta metall. 16, 95 (1982).

16. L. N. Chang, G. Taylor and J. W. Christian, Acta metall. 31, 37 (1983).

17. V. Vitek, Dislocations and Properties of Real Materials: Proc. Fiftieth Ann. Concept of the Dislocation in Crystals (edited by M. H. Loretto), Publ. 323, pp. 30-50. Inst. of Metals, London (1985)

18. M. S. Duesbery, R. Soc. Proc. A 392A, 145 (1984).

19. M. S. Duesbery, R. Soc. Proc. A 392A, 175 (1984).

20. V. Vitek, Crystal Latt. Defects 5, 1 (1974). 
21. F. Guiu, Scripta metall. 3, 449 (1969).

22. D. I. Boleff, J. appl. Phys. 32, 100 (1961).

23. D. I. Boleff and J. De Klerk, J. appl. Phys. 33, 2311 (1962).

24. F. Milstein and J. Marshall, Phil. Mag. 58A, 365 (1988).

25. G. L. Webb, T. E. Mitchell and R. Gibala, Metall. Trans. 5A, 581 (1974).

26. G. Taylor and C. F. Elam, R. Soc. Proc. 112A, 337 (1926).

27. K. J. Bowman and R. Gibala, Scripta metall. 20, 1451 (1987).

28. C. S. Kim, Y. W. Chung and M. E. Fine, Proc. Fatigue 87 (edited by R. O. Ritchie and E. A. Starke Jr), pp. 73-82. EMAS, West Midlands (1987).
29. D. Hull, J. F. Byron and F. W. Noble, Can. J. Phys. 45, 1091 (1967).

30. R. Lachenmann and H. Schultz, Scripta metall. 6, 731 (1972).

31. S. Takeuchi, E. Kuramoto and T. Suzuki, Acta metall. 20, 909 (1972).

32. K. J. Bowman et al., Proc. Eighth Int. Conf. on Strength of Metals and Alloys, Vol. 1, pp. 199-204. Pergamon Press, Oxford (1988).

33. M. Anglada and F. Guiu, Proc. Sixth Int. Conf. on Strength of Metals and Alloys, pp. 3-12. Pergamon Press, Oxford (1982).

34. M. Anglada and F. Guiu, Phil. Mag. 46A, 881.

35. F. Ackermann, H. Mughrabi and A. Seeger, Acta metall. 31, 1353 (1983). 